VINHETA IMAGENOLÓGICA

IMAGENOLOGIC VignETTE

Responsáveis: Artur da Rocha Corrêa Fernandes e Jamil Natour

\title{
Lúpus Eritematoso Sistêmico e Avaliação do Bem-Estar Fetal pela Dopplervelocimetria ${ }^{(*)}$ Systemic Lupus Erythematosus and Assessment
of Fetal Well-Being by Doppler Velocimetry
}

\author{
Nilson R. de Jesús(1), Roger A. Levy(2)
}

\section{INTRODUÇÃO}

O diagnóstico precoce do comprometimento fetal decorrente da insuficiente função placentária (reduzida troca materno-fetal), antes que ocorra dano ao encéfalo fetal, é hoje o principal objetivo da propedêutica de avaliação do bem-estar fetal. A avaliação da velocidade do fluxo sangüíneo feto-placentário e fetal representa um importante método de avaliação biofisica fetal nas gestações de alto risco que evoluem com vasculopatia, microangiopatia ou trombose. O lúpus eritematoso sistêmico (LES) é característico dessas gestações, principalmente quando associado à atividade da doença, à hipertensão arterial sistêmica (HAS), à nefrite ou à síndrome do anticorpo antifosfolipídeo (SAF). No Ambulatório Pré-Natal de Doenças Auto-Imunes do Hospital Universitário Pedro Ernesto, da Universidade do Estado do Rio de Janeiro, utilizamos a Dopplervelocimetria na avaliação do bem-estar fetal e no rastreamento precoce do sofrimento fetal crônico.

\section{CIRCULAÇÃO ÚTERO-PLACENTÁRIA E FETO- PLACENTÁRIA NA GESTAÇÃO NORMAL}

$\mathrm{Na}$ gestação normal as artérias espiraladas são responsáveis pelo aporte sangüíneo à decídua (endométrio gravídico) e ao espaço interviloso placentário. Logo nas primeiras semanas elas se tornam mais numerosas e tortuosas. No início do segundo trimestre o trofoblasto, em busca de nutrição, invade a capa musculoelástica dessas arteríolas, transformando-as em vasos calibrosos e de baixa resistência. Essa transformação vascular propicia maior aporte sangüíneo ao espaço interviloso, condição básica para nutrição e oxigenação fetal, e conseqüente desenvolvimento fetal adequado. Através das duas artérias umbilicais (AUs) cerca de 40\% do débito ventricular fetal atinge a circulação do sistema viloso terciário, que é banhado pelo sangue materno ejetado pelas artérias espiraladas no espaço interviloso (Figura 1). A avaliação da velocidade do fluxo sangüíneo na $A U$ fornece informações sobre a perfusão sangüínea da unidade feto-placentária. Com o crescimento da árvore vascular vilositária a resistência na AU diminui gradativamente no curso da gravidez. Desta forma, caracteristicamente, a onda de velocidade de fluxo (OVF) (Figura 2) na AU apresenta velocidade diastólica final elevada em decorrência da baixa resistência ao fluxo no território placentário (Figura 3). Ao contrário, na artéria cerebral média fetal (Figura 4) a resistência ao fluxo é elevada e, conseqüentemente, a velocidade diastólica final é baixa (Figura 5).

\footnotetext{
* Trabalho realizado na Faculdade de Ciências Médicas da Universidade do Estado do Rio de Janeiro (FCM-UERJ). Rio de Janeiro, RJ, Brasil.

1. Professor assistente, Disciplina de Obstetrícia. FCM-UERJ

2. Professor adjunto, Disciplina de Reumatologia. FCM-UERJ.
}

Endereço para correspondência: Prof. Nilson R. de Jesús. Blvd 28 de setembro 77, sala 441. Vila Isabel, Rio de Janeiro, RJ. Tel. (21) 2587-6825. 


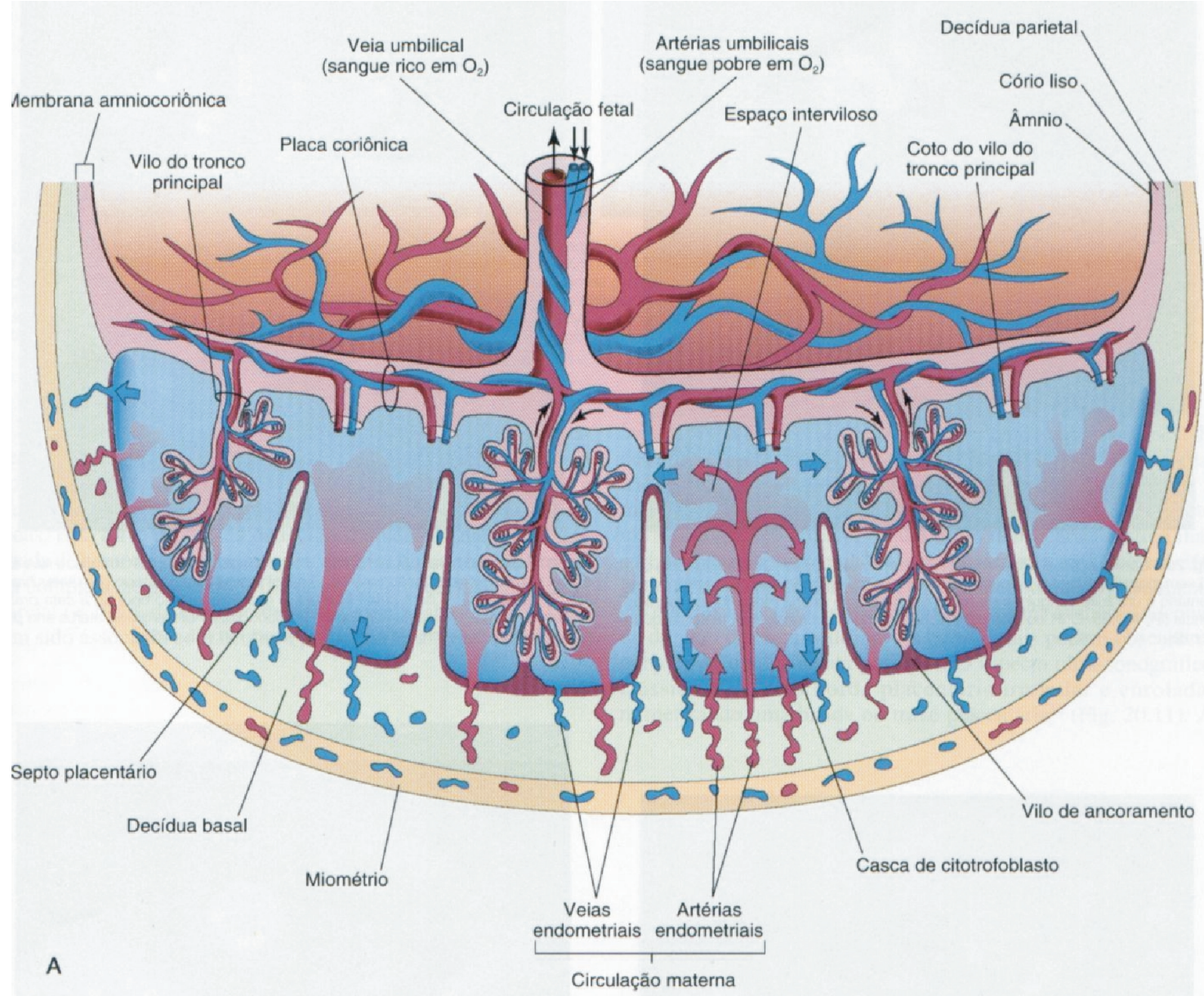

FIgURA 1 - Diagrama esquemático da placenta, no qual pode-se observar a relação entre a circulação fetal, a vilosidade corial, o espaço interviloso e as artérias espiraladas (artérias endometriais). Adaptado de Callen PW. Ultrasonography in obstetrics and gynecology. 4th ed.

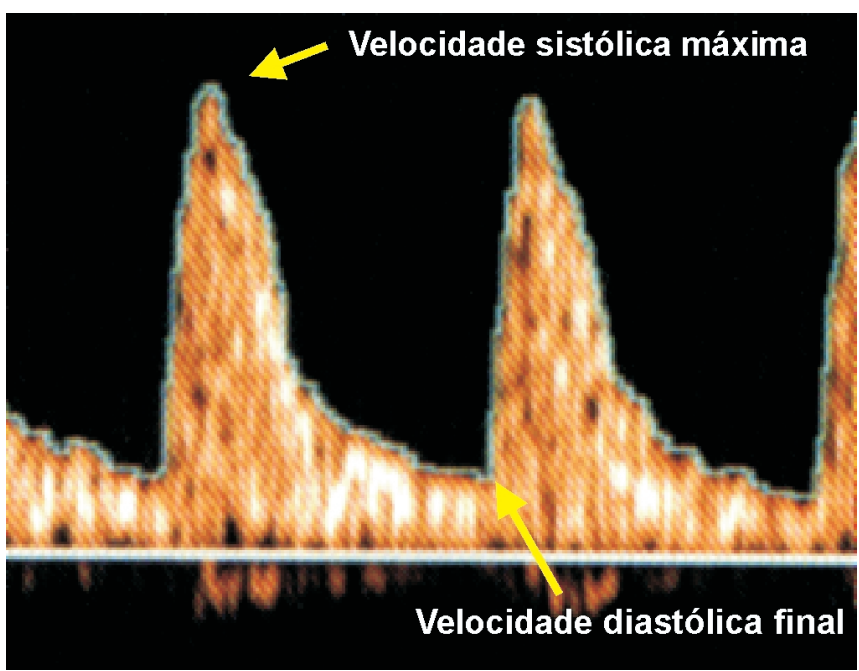

Figura 2 - Onda de velocidade de fluxo

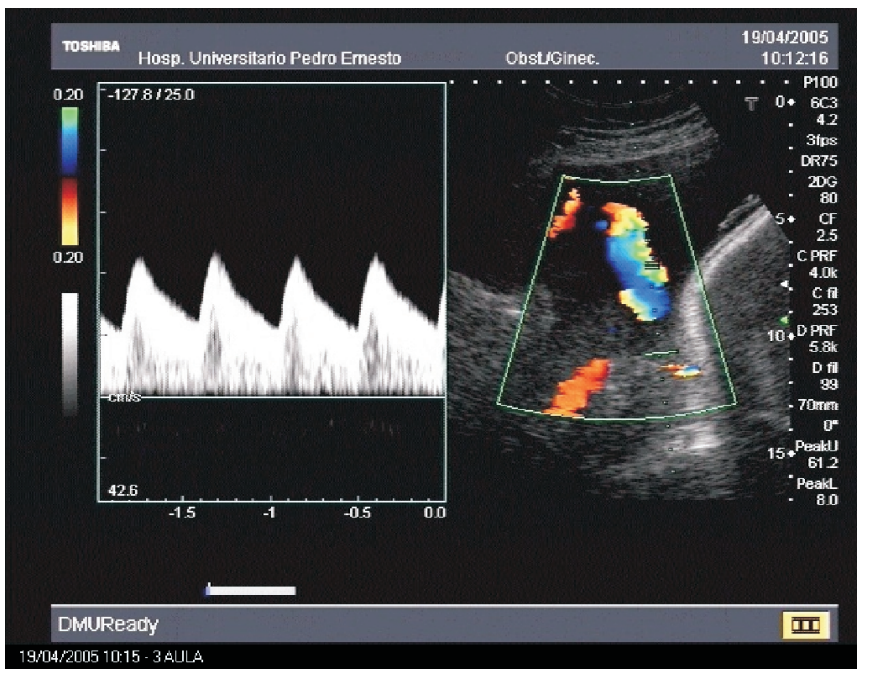

FiguRA 3 - Doppler da artéria umbilical com fluxo normal 


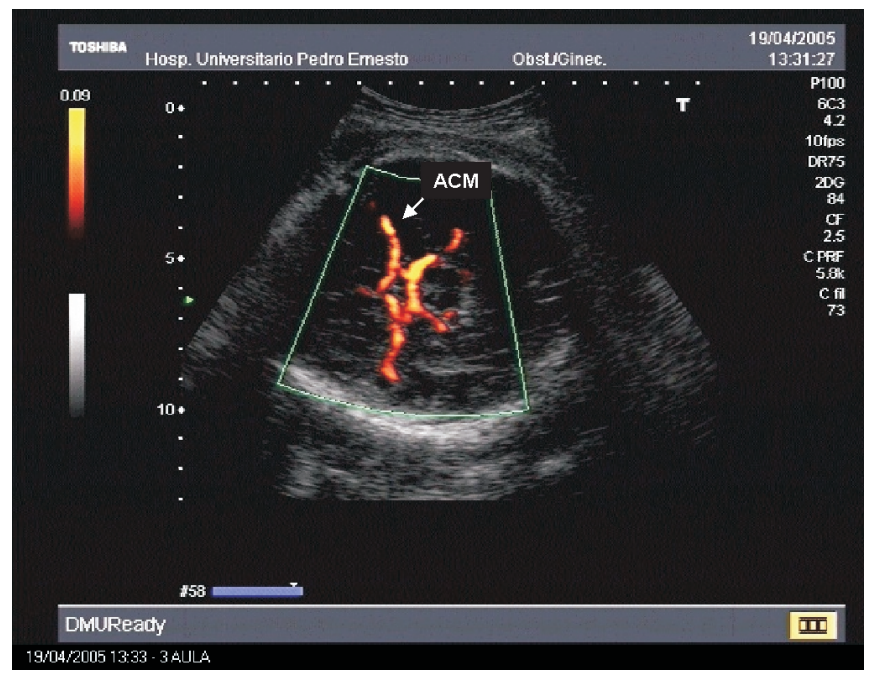

Figura 4 - Doppler colorido das artérias cerebrais médias. Identifica-se ainda o polígono de Willis e as artérias cerebrais posteriores

\section{ALTERAÇÕES NAS DOENÇAS QUE CAUSAM VASCULOPATIA MATERNA}

O LES, quer seja primariamente quer associado à HAS, nefrite ou SAF, compromete o bem-estar fetal, resultando, na maioria dos estudos, em elevadas taxas de sofrimento fetal crônico, restrição do crescimento intra-uterino (CIUR), prematuridade e óbito fetal tardio. As lesões vasculares obstrutivas deciduais conduzem a um precário desenvolvimento da massa placentária, repercutindo de modo importante no crescimento vilositário, caracterizando o mecanismo de "má placentação". O impedimento do fluxo sangüíneo normal através do sistema vilositário resulta em aumento da resistência na AU e retorno ao feto de sangue mal oxigenado.

\section{CENTRALIZAÇÃO}

Submetido a um regime de hipoxemia prolongado, o feto desencadeia um mecanismo de defesa redistribuindo o sangue através de vasoconstrição e vasodilatação seletiva. No esforço de priorizar a oxigenação de órgãos nobres, como cérebro, coração e supra-renais, o feto promove vasodilatação nesses territórios e vasoconstrição nos tecidos adiposo e muscular, e vísceras. Como resultado, encontraremos um feto com CIUR assimétrico (pólo cefálico normal e corpo com crescimento restrito). Nesse momento, com mais de 50\% do sistema vilositário obstruído, a avaliação pela Dopplervelocimetria demonstra inversão da relação

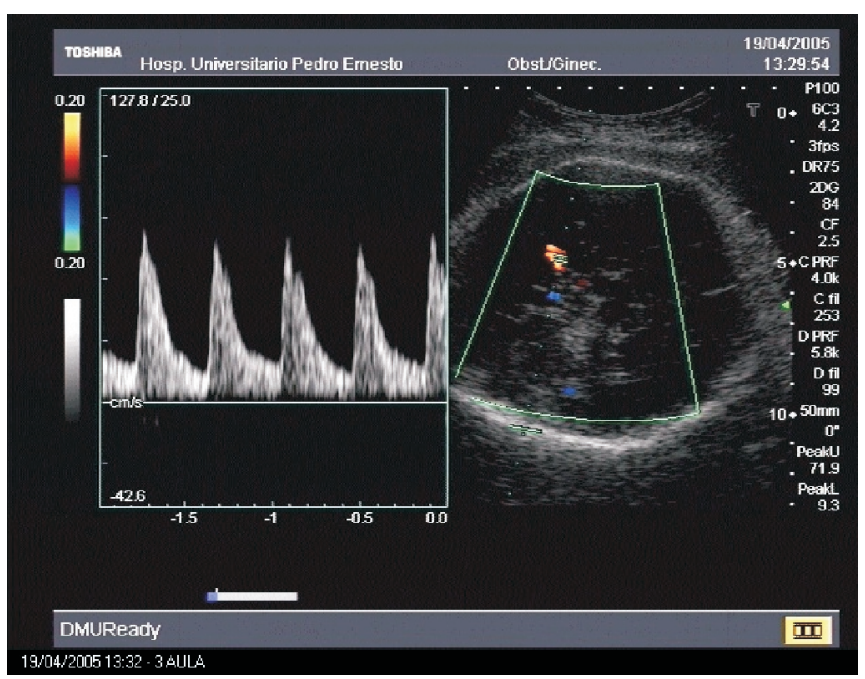

FiguRA 5 - Doppler da artéria cerebral média com fluxo normal

entre os fluxos da AU e da artéria cerebral média (ACM), ou seja, AU com fluxo de alta resistência (Figura 6) e ACM com resistência diminuída (Figura 7). O diagnóstico de centralização é feito quando a relação umbílico-cerebral é igual ou maior que 1 . Recentes observações correlacionam a centralização com a presença do fator de necrose tumoral (TNF) e de interleucina-6 (IL-6) na circulação materna. Tal fato poderia ser reflexo precoce de dano cerebral fetal ou, simplesmente, secundário à lesão placentária.

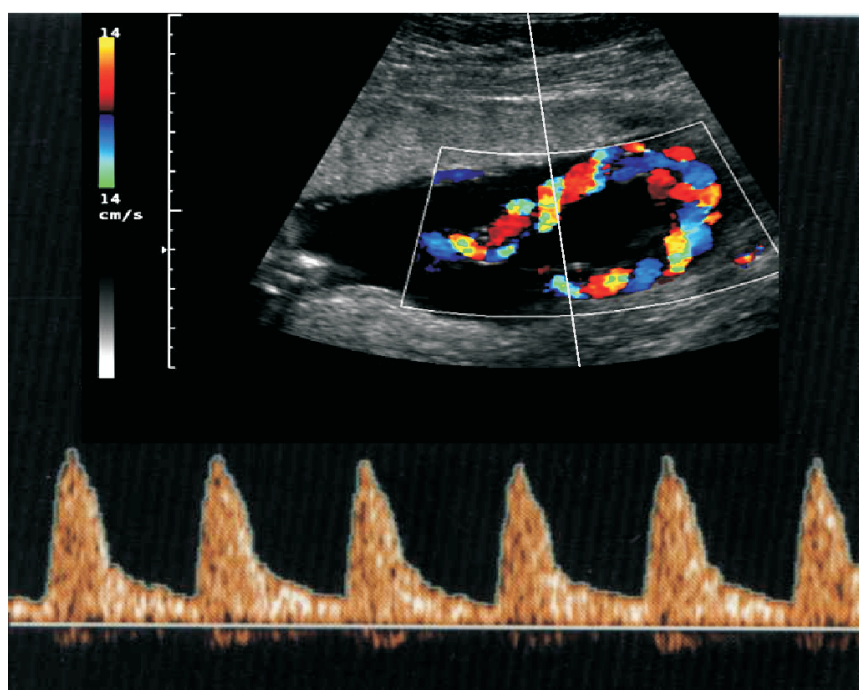

FIGURA 6 - Doppler da artéria umbilical com fluxo alterado de alta resistência 


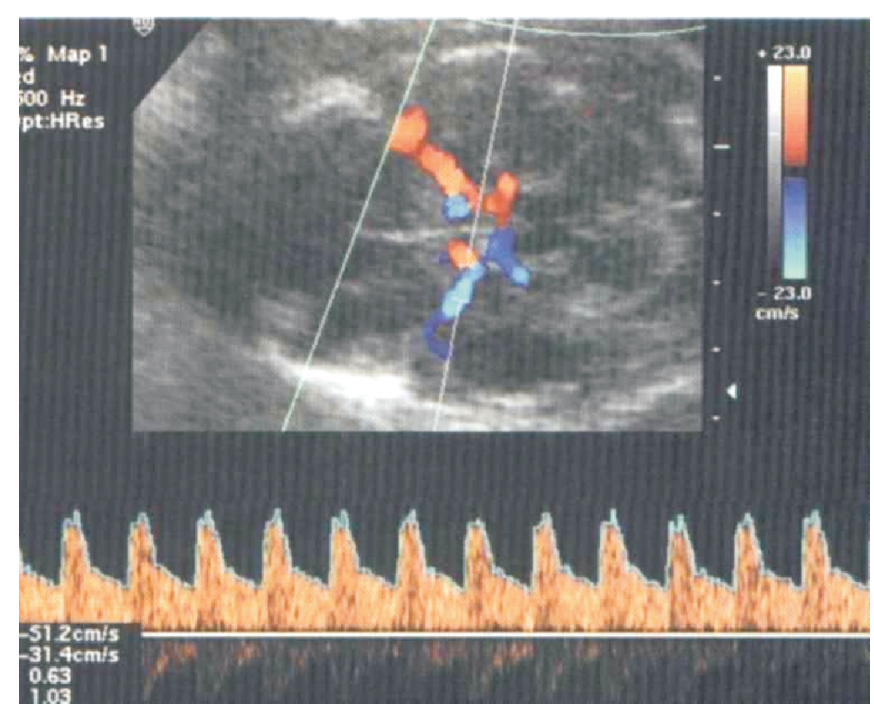

FiguRa 7 -Doppler da artéria cerebral média com fluxo alterado de baixa resistência

\section{DIÁSTOLE ZERO E DIÁSTOLE REVERSA}

Prolongando-se a gravidez do feto que já apresenta centralização, a resistência ao fluxo na AU continua aumentando até chegar à diástole zero, isto é, durante a diástole cardíaca fetal o sangue não circula (Figura 8). Quando pela Dopplervelocimetria se diagnostica a diástole zero, cerca de $90 \%$ dos vasos vilositários estão obstruídos. Estágio mais grave se segue quando durante a diástole ocorre fluxo retrógrado, caracterizando a diástole reversa (Figura 9).

\section{CONDUTA NA ASSISTÊNCIA ÀS GESTANTES PORTADORAS DE LES}

Utilizando-se como orientação os elevados índices de morbidade e mortalidade fetal nas gestantes portadoras de LES realizamos, em nosso ambulatório pré-natal, monitoração intensiva do bem-estar fetal prioritariamente por meio da Dopplervelocimetria. Essa avaliação é desencadeada a partir da $26^{\text {a }}$ semana e repetida, se normal, a cada quatro semanas. Quando a centralização é detectada prescrevemos a corticoterapia com betametasona para a prevenção da síndrome de membrana hialina e programamos a antecipação do parto. Na ocorrência de diástole zero ou reversa a antecipação do parto é imediata.

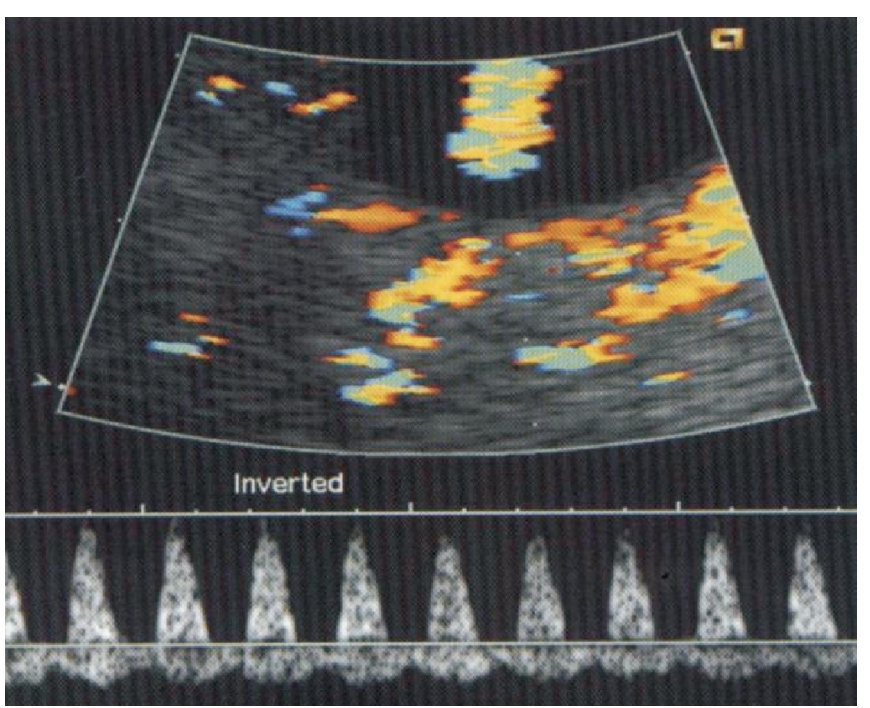

FIgURA 8 -Doppler da artéria umbilical com diástole zero

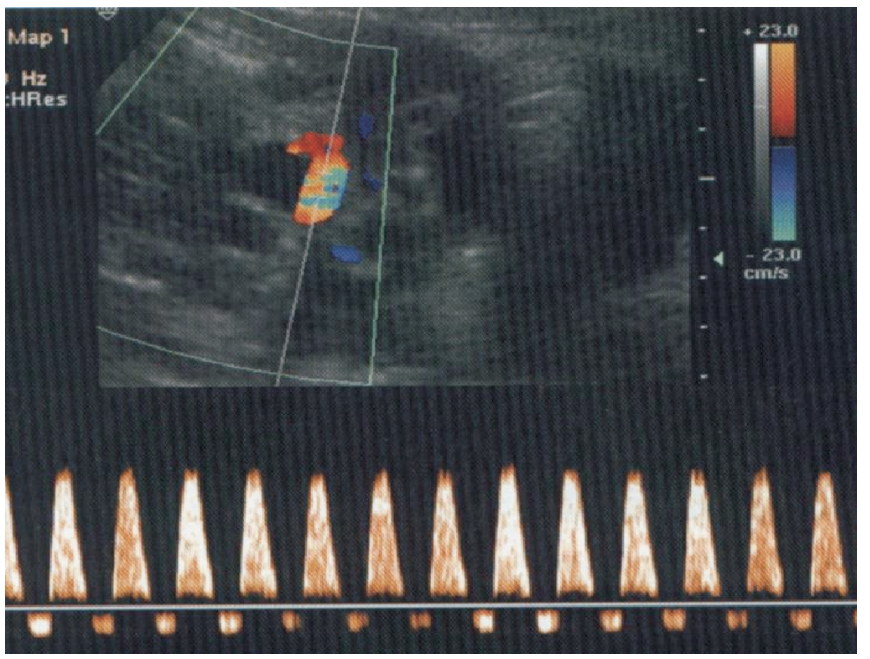

Figura 9 -Doppler da artéria umbilical com diástole reversa

A Dopplervelocimetria representa atualmente importante instrumento propedêutico, que permite avaliar com segurança o bem-estar fetal, evitando-se com antecedência que estágios de asfixia grave, como diástole zero e reversa, sejam atingidos e possibilitando que o recém-nascido prematuro submetido ao cuidado do neonatologista apresente baixo risco de complicações neonatais. 
Jesús et al.

\section{REFERÊNCIAS}

1. Callen PW: Ultrasonography in obstetrics and gynecology. 4th ed. Philadelphia, W B Saunders Company, 2000.

2. Dubiel M, Seremak-Mrozikiewicz A, Breborowicz GH, Drews K, Pietryga M, Gudmundsson S: Fetal and maternal Doppler velocimetry and cytokines in high-risk pregnancy. J Perinat Med 33: 17-21, 2005.
3. Farine D, Granovsky-Grisaru S, Ryan G, et al: Umbilical artery blood flow velocity in pregnancies complicated by systemic lupus erythematosus. J Clin Ultrasound 26: 379-382, 1998.

4. Harkness UF, Mari G: Diagnosis and management of intrauterine growth restriction. Clin Perinatol 31: 743-64, 2004.

5. Kitridou RC, Goodwin TM: The fetus in systemic lupus erythematosus. In: Dubois' lupus erythematosus. 6th ed. Philadelphia, Lippincot Williams \& Wilkins, 2002. 